\title{
Análise dos elementos metálicos no mel como uma ferramenta para o monitoramento ambiental
}

\author{
Analysis of metals in honey for environmental monitoring
}

\author{
Ketelen Michele Guilherme de Oliveira ${ }^{1}\left({ }^{*}\right)$ \\ Lucila Akiko Nagashima²
}

\section{Resumo}

O objetivo deste trabalho foi efetuar a caracterização dos elementos metálicos $\mathrm{Mg}, \mathrm{Ca}$, $\mathrm{K}, \mathrm{Zn}, \mathrm{Pb}, \mathrm{Na}, \mathrm{Co}, \mathrm{Fe}, \mathrm{Cu}$ e $\mathrm{Mn}$ em amostras de mel provenientes da região noroeste do Paraná, pela Espectrometria de Absorção Atômica de Chama. As análises foram efetuadas no Laboratório de Química - Departamento de Química da Universidade Estadual de Maringá, Paraná. Foram analisadas onze amostras, das quais três apresentaram níveis do metal chumbo elevado, quando comparadas com o máximo permitido pela legislação brasileira. A detecção desse elemento (chumbo) e outros metais pesados em grandes proporções podem indicar que o ambiente em que se encontra a colmeia está contaminado. Diversos são os vínculos de contaminação, desses, solo, ar, água, e até materiais utilizados na área de extração do mel, podem consequentemente, serem introduzidos juntos na colmeia.

Palavras-chave: metais pesados; biomonitoramento; mel.

\section{Abstract}

The objective of this work was to characterize the metals $\mathrm{Mg}, \mathrm{Ca}, \mathrm{K}, \mathrm{Zn}, \mathrm{Pb}, \mathrm{Na}, \mathrm{Co}, \mathrm{Fe}$, $\mathrm{Cu}$ and $\mathrm{Mn}$ in honey samples from the northwestern region of Paraná State, Brazil. by Atomic Absorption Spectrometry Flame. The samples were analyzed at the Chemistry Laboratory - Department of Chemistry, Universidade Estadual de Maringá, Paraná. Eleven samples was analyzed, three of which had high levels of the metal lead, compared to the maximum allowed by Brazilian law. The detection of this element $(\mathrm{Pb})$ and other heavy metals in major proportion, may indicate that the environment in which the honeycomb is contaminated located. There are several links contamination, these soil, air, water, and even materials used in honey extraction area, may accordingly be adhered to the surface of the bee's body, and are introduced together in the hive.

Key words: heavy metals; biomonitoring; honey.

1 Mestranda; Genética; Universidade Federal do Paraná, UFPR, Brasil; Desenvolve Iniciação Cientifica na Universidade Estadual do Paraná/Campus de Paranavaí, UNESPAR, Brasil; Endereço: Universidade Federal do Paraná. ACF Centro Politécnico, Jardim das Américas 81531980 - Curitiba, PR - Brasil; E-mail: ketelenguioliveira@hotmail.com (*) Autor para correspondências

2 Dra.; Engenharia Química; Universidade Estadual de Maringá, UEM, Brasil; Professor adjunto da Universidade Estadual do Paraná; Endereço: Faculdade Estadual de Educação Ciências e Letras de Paranavaí. Avenida Gabriel Esperidião, s/n Jardim Morumbi, 87703-000 - Paranavai, PR - Brasil; E-mail: ucilanagashima@uol.com.br

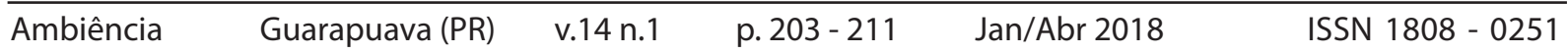




\section{Introdução}

O mel possui, em sua composição, diversos minerais que são importantes para o metabolismo humano. Esses minerais são, em parte, provenientes do solo, que são absorvidos pelas plantas e transportados para o néctar. Dessa forma, as características e composição do mel variam de acordo com os tipos de solos e com a origem floral que é utilizada pelas abelhas (EPIFÂNIO, 2012).

Em busca dos materiais necessários para sua alimentação, as abelhas percorrem uma área de até $7 \mathrm{~km}$, e que cerca o seu habitat. Elas usufruem de todos os compartimentos ambientais, como o solo, as plantas, o ar e, também, a água. A poluição desses compartimentos com metais pesados (Figura 1), microrganismos, produtos químicos e partículas em suspensão no ar, poluentes atmosféricos (escapamentos de automóveis, de chaminés de fábricas, siderúrgicas, minerações, depósitos de lixo, entre outros), são interceptados pelas abelhas e podem ficar armazenados na superfície do seu corpo e introduzidos nas colmeias juntamente com o pólen (RIBEIRO, 2010; PORRINI et al., 2003). A contaminação ainda pode ocorrer por meio do néctar, do pólen, da melada, ou da água ingerida.

\section{Figura 1. Contaminação do ambiente com metais pesados, a área circulada representa os compartimentos visitados pelas abelhas}

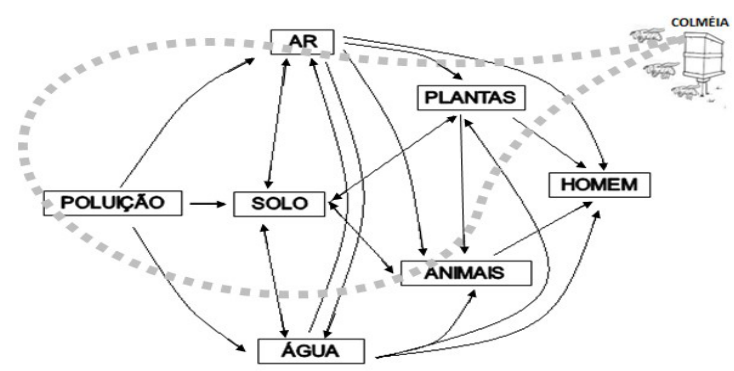

Fonte: Adaptado de Porrini et al. (2003).

Os metais pesados, quando em excesso, são potencialmente prejudiciais, podendo atuar como elementos mutagênicos, carcinogênicos e citotóxicos ao nosso organismo. Esses elementos quando presentes no néctar das flores, permanecem nele, inclusive após sua transformação pelas abelhas, em mel. Metais pesados, presentes nos produtos apícolas, em níveis acima dos estabelecidos por legislações pertinentes, representam ameaça para os seres humanos em função dos efeitos negativos e cumulativos de tais contaminantes para o organismo. O rim e o sistema nervoso central são órgãos muito sensíveis aos metais pesados. Uma exposição alterada a esses elementos poderia levar à insuficiência renal (BURBURE et al., 2006).

Os metais pesados são persistentes no ambiente, pois não podem ser degradados e, consequentemente, acumulam com o tempo. Os elementos $\mathrm{Cu}, \mathrm{Zn}$ e $\mathrm{Pb}$ são altamente perigosos, devido ao seu potencial de toxicidade e à capacidade de acumulação no solo (OLIVEIRA et al., 2010).

Pautado nesse acontecimento, as abelhas e seus produtos podem ser usados como bioindicadores para monitoramento de impacto ambiental provocado pelos fatores físicos, químicos e biológicos (RISSATO et al., 2006). Assim, a concentração dos metais traço nos 
produtos apícolas reproduz o perfil de metais de toda a região visitada pelas abelhas operárias, sendo excelentes bioindicadores ambientais, útil na avaliação do grau de contaminação daquele espaço. No Brasil, há legislações que estabelece o limite máximo de contaminantes nos alimentos, tais como o Decreto 55.871/1965 do Ministério da Saúde, Portaria 11/1987 da Secretaria Nacional de Vigilância Sanitária, Portaria 685/1998, Instrução Normativa 42/1999, o anexo IV da Instrução Normativa 14/2009 que consiste no programa de controle de resíduos e contaminantes do mel (BRASIL, 2009).

\section{Tabela 1. Concentração máxima para alguns metais em mel}

\begin{tabular}{ccccc}
\hline & \multicolumn{4}{c}{ Concentração máxima permitida $\left(\boldsymbol{\mu g . g ^ { - 1 }}\right)$} \\
\cline { 2 - 5 } Elementos & $\begin{array}{c}\text { Dec. } \\
\mathbf{5 5 8 7 1 / 6 5}\end{array}$ & Port. 11/87 & Port. 685/98 & Inst. Norm. 14/09 \\
\hline Chumbo $(\mathrm{Pb})$ & 0,80 & Não definido & Não definido & 0,50 \\
Cobre $(\mathrm{Cu})$ & 30,0 & Não definido & 10,00 & Não definido \\
Zinco $(\mathrm{Zn})$ & 50,00 & Não definido & Não definido & Não definido \\
\hline
\end{tabular}

Fontes: Decreto 55871/65 (BRASIL, 1965). Portaria 11/87 (BRASIL, 1987). Portaria 685/98 (BRASIL, 1998). Instrução Normativa 14/09 (BRASIL, 2009).

Diante disso, o objetivo do trabalho foi a determinação da concentração dos elementos traço $\mathrm{Mg}, \mathrm{Ca}, \mathrm{K}, \mathrm{Zn}, \mathrm{Pb}, \mathrm{Na}, \mathrm{Co}, \mathrm{Fe}, \mathrm{Cu}$ e Mn, pela Espectrometria de Absorção Atômica de Chama, em amostras de mel comercializadas na cidade de Paranavaí, Estado do Paraná.

\section{Materiais}

Foram pesquisadas onze variedades de mel comercializadas na cidade de Paranavaí, Estado do Paraná. Na Tabela 2, estão discriminadas as características das amostras empregadas na determinação dos metais.

Tabela 2. Amostras de mel e sua origem.

\begin{tabular}{cccc}
\hline Códigos & Fonte fornecedora & Origem floral & Período de produção \\
\hline Mel A & Apiário & Silvestre & Setembro de 2013 \\
Mel B & Comercializado & Silvestre & Novembro de 2013 \\
Mel C & Apiário & Silvestre & Dezembro de 2013 \\
Mel D & Comercializado & Silvestre & Janeiro de 2014 \\
Mel E & Apiário & Laranjeira & Janeiro de 2014 \\
Mel F & Apiário & Silvestre & Setembro de 2013 \\
Mel G & Comercializado & Silvestre & Julho de 2013 \\
Mel H & Apiário & Silvestre & Setembro de 2013 \\
Mel I & Apiário & Silvestre & Dezembro de 2013 \\
Mel J & Apiário & Laranjeira & Janeiro de 2014 \\
\hline
\end{tabular}

Fontes: Autores (2015).

As vidrarias empregadas nessa atividade foram previamente lavadas com $\mathrm{HCl} 10 \%$ para remoção dos contaminantes presentes, e enxaguadas com água destilada. As amostras de mel para a determinação do $\mathrm{Mg}, \mathrm{Ca}, \mathrm{K}, \mathrm{Zn}, \mathrm{Pb}, \mathrm{Na}, \mathrm{Co}, \mathrm{Fe}, \mathrm{Cu}$ e $\mathrm{Mn}$ foram pré-concentradas, por OLIVEIRA, K. M. G. et al. 
meio de aquecimento em chapa elétrica, a temperatura de $60^{\circ} \mathrm{C}$, a fim de assegurar concentração metálica suficiente para a determinação, em função do limite de detecção imposto pela técnica de Espectrometria de Absorção Atômica de Chama (EAA). As condições de operação variaram para cada metal pesquisado. As condições ótimas para determinação dos múltiplos elementos foram estabelecidas conforme as recomendações do fabricante do equipamento. Em um béquer, foram adicionadas $2 \mathrm{~g}$ de mel, $4 \mathrm{~mL}$ de ácido nítrico $\left(\mathrm{HNO}_{3}\right) \mathrm{PA}$ e água destilada até completar o volume de $100 \mathrm{~mL}$ e submetido ao aquecimento para a digestão do material. A seguir o material foi filtrado, estocado em um frasco lavado com $\mathrm{HCl} \mathrm{10 \%} \mathrm{e} \mathrm{armazenado} \mathrm{sob} \mathrm{refrigeração}$ até a determinação dos níveis de metais. As análises dos elementos metálicos foram efetuadas no Departamento de Química da Universidade Estadual de Maringá pela Espectrometria de Absorção Atômica, segundo metodologia da Association of Official Analytical Chemitrys.

\section{Resultados e Discussão}

$\mathrm{Na}$ tabela 3, são apresentados os valores médios em $\left(\mathrm{mg} \cdot \mathrm{kg}^{-1}\right)$ para os elementos analisados. O metal Co não foi detectado nas amostras. Observou-se variabilidade na concentração dos minerais na composição das amostras pesquisadas.

Tabela 3. Níveis de metais detectados nas amostras de mel

\begin{tabular}{|c|c|c|c|c|c|c|c|c|c|c|}
\hline \multirow{2}{*}{ Amostras } & \multicolumn{10}{|c|}{ Média dos valores obtidos (mg. $\left.\mathrm{kg}^{-1}\right)$} \\
\hline & $\mathrm{Mg}$ & $\mathrm{Ca}$ & $\mathbf{K}$ & $Z_{\mathbf{n}}$ & $\mathbf{P b}$ & $\mathrm{Na}$ & Co & $\mathbf{F e}$ & $\mathrm{Cu}$ & Mn \\
\hline Mel A & 70,12 & 52,91 & 651,52 & 2,76 & 2,57 & 84 & nd & nd & 0,81 & 7,11 \\
\hline Mel B & 29,32 & $\mathrm{nd}^{*}$ & 527,84 & 3,60 & 4,08 & 82,17 & nd & nd & 1,27 & 2,07 \\
\hline Mel C & 68,85 & 14,75 & 1583,54 & 1,18 & nd & 87,23 & nd & nd & 0,81 & 2,35 \\
\hline Mel D & 26,77 & nd & 493,42 & 0,78 & nd & 87,62 & nd & nd & 0,58 & 2,32 \\
\hline Mel E & 40,80 & nd & 750,33 & 1,23 & 0,87 & 76,11 & nd & nd & 0,43 & 2,66 \\
\hline Mel F & 40,12 & nd & 1528,72 & 0,98 & nd & 87,53 & nd & nd & 0,93 & 9,86 \\
\hline Mel G & 194,4 & 623,4 & 1711,0 & 1,3 & nd & 184,8 & nd & 0,8 & 1,8 & 5,3 \\
\hline Mel H & 265,2 & 677,0 & 2564,0 & 1,2 & nd & 247,3 & nd & 0,8 & 2,5 & 8,0 \\
\hline Mel I & 326,4 & 810,9 & 2926,7 & 1,1 & nd & 226,3 & nd & 1,0 & 1,4 & 13,1 \\
\hline Mel J & 275,4 & 740,7 & 2717,6 & 0,7 & nd & 193,1 & nd & 1,2 & 1,7 & 9,8 \\
\hline Mel K & 242,2 & 780,3 & 2354,2 & 0,4 & nd & 149,8 & nd & nd & 1,5 & 6,8 \\
\hline $\begin{array}{l}\text { Nível de } \\
\text { detecção }\end{array}$ & - & - & - & - & 0,03 & - & - & 0,04 & - & 0,02 \\
\hline
\end{tabular}

Fontes: Autores (2015).

Nota: nd $^{*}=$ não detectado

Os elementos $\mathrm{Na}, \mathrm{K}, \mathrm{Ca}, \mathrm{Fe}, \mathrm{Cu}, \mathrm{Mg}$ e $\mathrm{Zn}$ são denominados como elementos essenciais, pois são necessários ao metabolismo biológico dos organismos vivos, entretanto em nível traço, da ordem de miligrama (RIBEIRO,2010). Já o elemento Pb é classificado como micro-contaminante ambiental e não necessário ao organismo vivo em nenhuma quantidade. $\mathrm{O} Z \mathrm{n}, \mathrm{Fe}$ e $\mathrm{Mn}$ são também micro-contaminantes, porém necessários ao organismo vivo, na ordem de microgramananograma, entretanto acima desses níveis, esses metais podem se tornar potencialmente tóxicos. 
A planta também utiliza os minerais da solução do solo para sua nutrição e equilíbrio do seu crescimento vegetativo e reprodutivo. Os nutrientes minerais essenciais para a planta são divididos em nutrientes orgânicos, macronutrientes, micronutrientes e elementos úteis como o sódio, por exemplo. Potássio, cálcio, e magnésio estão entre os minerais exigidos em grandes quantidades pelas plantas, sendo denominados macronutrientes. A deficiência do potássio por exemplo, causa interferência na síntese proteica, retardando a maturação, e produzindo frutos verdes, duros e ácidos. Os elementos cobre, ferro, manganês e zinco são exigidos em pequenas quantidades e são chamados de micronutrientes (ALBUQUERQUE, 2004). Dessa forma, os minerais absorvidos do solo serão transportados para o néctar coletado pela abelha, e para os animais e ao homem na forma de forragem ou alimento.

Os metais pesados, quando em excesso, exercem efeitos negativos sobre o crescimento das plantas, e também afetam os processos bioquímicos que ocorrem no solo. A decomposição do material orgânico adicionado ao solo, a mineralização do nitrogênio e a nitrificação podem ser inibidos em locais contaminados por metais pesados. (TSUTIYA, 1999)

No ambiente, encontramos metais pesados em forma biodisponíveis para os seres vivos, podendo estar nas formas solúveis, em que o metal está na forma iônica e pode ser facilmente absorvido pelas plantas; trocáveis, na qual o metal ligado eletrostaticamente em sítios de adsorção carregados negativamente na matéria orgânica ou nas argilas, sendo facilmente trocado por íons presentes na solução do solo. Essas formas são as mais preocupantes, pois apresentam maior biodisponibilidade. Ainda podem ser encontrados nas formas de precipitado, adsorvidos especificamente, e ligados a materiais orgânicos insolúveis. (MEURER, 2004)

Os teores dos metais não apresentaram homogeneidade, devido às amostras serem multiflorais, característico de mel silvestre. No Brasil, o mel é principalmente de origem silvestre, devido ao fato de que as florestas brasileiras possuem uma grande quantidade de diferentes espécies. O intervalo de variação foi elevado, com exceção para o ferro que variou de 0,8 a $1,2 \mathrm{mg} \cdot \mathrm{kg}^{-}$ ${ }^{1}$.O potássio, o cálcio, o magnésio e o sódio foram os elementos mais abundantes encontrados nas amostras, o cálcio porém não foi detectado nas amostras de Mel B, D, E e F. Encontrou-se conformidade com vários autores como BERTOLDI el al., 2010; SANTOS et al., 2008; SODRÉ et al., 2007, as quais verificaram maiores níveis para esses minerais nas amostras de mel. O potássio e o cálcio apresentaram concentrações variando de 493,42 a 2926,7mg. $\mathrm{kg}^{-1}$ e 14,75 a 810,9mg. $\mathrm{kg}^{-1}$, respectivamente. Valores próximos foram encontrados por Bertoldi et al. (2010), sendo que o potássio variou de 262,96 a 1767,67mg. $\mathrm{kg}^{-1}$ e o cálcio de 30,35 a 184,20 mg. $\mathrm{kg}^{-1}$. Nas amostras de mel, também foram detectadas grandes quantidades dos minerais magnésio (26,77 a 326,4mg. $\mathrm{kg}^{-1}$ ) e sódio $\left(76,11 \mathrm{a} 247,3 \mathrm{mg} \cdot \mathrm{kg}^{-1}\right)$. Santos et al. (2008), encontraram para esses metais uma variação de 5,38 a 33,44 mg. $\mathrm{kg}^{-1}$ e 21,87 a 840,40 mg. $\mathrm{kg}^{-1}$, respectivamente. A quantidade de minerais está associada com a cor do mel, sendo que, quanto maior a quantidade desses elementos presentes nas amostras, mais escura é a sua coloração. O consumidor tem preferência por produtos com coloração mais clara, sendo atribuído, então, para estes, o preço mais elevado.

Os metais pesados se encontram distribuídos por toda a natureza. Os elementos $\mathrm{Cu}, \mathrm{Pb}$ e $\mathrm{Zn}$, estão entre os metais mais fortemente adsorvidos e têm tempo de residência de 1000 a 3000 anos. Esses metais têm aumentado em ambientes de complexos industriais, e em áreas rurais de agricultura altamente tecnificada. A atividade mineradora também é um forte mecanismo de disponibilização de metais. Nos solos, os metais podem ser originados a partir da rocha de origem e de outras fontes adicionadas ao solo. Eles podem ser acumulados no solo pela deposição atmosférica, cinzas, calcário, fertilizantes químicos e adubos orgânicos, e lixo domiciliar o qual pode apresentar principalmente cádmio, cobre, chumbo e zinco. 
A retenção dos metais pesados e a vulnerabilidade a essa contaminação no solo, é proveniente de fenômenos como a capacidade de troca catiônica, área superficial específica, teores de óxidos (ferro, alumínio e manganês), $\mathrm{pH}$, teor de matéria orgânica, teor de argila, profundidade dos solos, tipologia das argilas, entre outros.

A concentração do zinco variou entre 0,4 a $3,60 \mathrm{mg} \cdot \mathrm{kg}^{-1}$, valores dentro dos limites observados por Hernández et al. (2004), cujo valores máximos e mínimos foram 0,18 a 19,1mg. $\mathrm{kg}^{-1}$, respectivamente. $\mathrm{O}$ mineral $\mathrm{Mn}$ foi detectado em todas as amostras, sendo que a menor concentração foi obtida no Mel B (2,07 mg. $\left.\mathrm{kg}^{-1}\right)$ e a mais elevada foi encontrada no Mel I $(13,11$ $\left.\mathrm{mg} \cdot \mathrm{kg}^{-1}\right)$, valores mais elevados quando comparados com a pesquisa efetuada por Conti et al. (2014), em amostras de mel multifloral, provenientes da Argentina, cujos resultados variaram de 0,14 a $3,13 \mathrm{mg} \cdot \mathrm{kg}^{-1}$.

O cobre apresentou nível mínimo de $0,43 \mathrm{mg} \cdot \mathrm{kg}^{-1}$ no mel E e máximo de $2,5 \mathrm{mg} \cdot \mathrm{kg}^{-1}$ para o mel H. Sodré et al., (2007), ao caracterizar elementos metálicos no mel de abelhas africanizadas do Estado do Piauí, observou uma variação de 0,007 a 0,908 mg. $\mathrm{kg}^{-1}$ a partir de 38 amostras analisadas. Em pesquisa realizada na Alemanha, o pesquisador Raeymaekers (2006) encontrou uma concentração de cobre variando de 0,04 a 1,0 mg. $\mathrm{kg}^{-1}$, cujos níveis são inferiores aos valores obtidos nesta pesquisa.

O metal chumbo $(\mathrm{Pb})$ foi detectado na amostra de mel $\mathrm{A}, \mathrm{B}$ e $\mathrm{E}$, sendo que a amostra $\mathrm{B}$ apresentou o nível mais elevado, cuja concentração foi de $4,08 \mathrm{mg} \cdot \mathrm{kg}^{-1}$. Assim, observou-se que o teor de $\mathrm{Pb}$ detectado nas amostras $\mathrm{A}, \mathrm{B}$ e $\mathrm{E}$ foi superior aos limites determinados pelo Decreto 55871/65 e pela Instrução Normativa, cujos valores estabelecidos são 0,80 mg. $\mathrm{kg}^{-1}$ e 0,50 mg. $\mathrm{kg}^{-1}$, respectivamente. Foi superior também aos níveis obtidos por Celechovska et al. (2001), cujos valores foram de 0,02 a 1,0 mg. $\mathrm{kg}^{-1}$. Já os níveis de $\mathrm{Cu}$ e $\mathrm{Zn}$ estão dentro dos limites estabelecidos pelas legislações contidas na Tabela 1 .

A presença de $\mathrm{Pb}$ em níveis acima dos limites estabelecidos pelas legislações (Tabela 1) podem ter sido resultantes de práticas apícolas indesejadas como as colmeias pintadas com tintas e vernizes para conservação das mesmas (RIBEIRO,2010). O estado de conservação das colmeias é importante, sendo necessária a pintura para a sua renovação. No entanto, é importante dar atenção aos produtos usados para essa finalidade. As tintas e os vernizes protetores da madeira podem ser a principal fonte de perigos de natureza química, contendo a presença de pesticidas ou metais pesados nas tintas.

Outra hipótese seria a localização das colmeias em regiões ricas em indústrias metalúrgicas e químicas que, reconhecidamente, contribuem para a contaminação do ambiente, como é o caso do mel A e B. Já a colmeia do mel B está localizada na região onde há produção agrícola e, provavelmente, o nível de chumbo pode ser proveniente do uso de inseticida na região, uma vez que o arsenato de chumbo pode ser um dos componentes do inseticida. Uma outra fonte de chumbo poderia ser o solo que é considerado um dos principais depósitos do metal. Assim, pode-se afirmar que os produtos apícolas passam por processo de bioacumulação, sendo muito útil na coleta de informações relacionadas ao ambiente onde as abelhas vivem e coletam o pólen e o néctar para a síntese do mel. Durante esse processo de coleta do material, as abelhas podem interceptar partículas suspensas no ar, estas ficarem armazenadas na superfície do seu corpo e serem depositadas na colmeia junto com o pólen, ou podem ser absorvidas junto com o néctar das flores, ocasionando a contaminação do produto. 
A contaminação com metais pesados pode também ocorrer durante o processo de produção do mel, por meio da utilização de materiais inadequados pelos apicultores, nas áreas de extração e armazenamento. A alimentação artificial é importante para manter os enxames com tamanhos médios, na entressafra, porém, alguns elementos metálicos podem ser introduzidos na alimentação artificial das abelhas, como xarope de açúcar orgânico ou de melado de açúcar orgânico. Estes podem apresentar elevadas concentrações de $\mathrm{Cd}, \mathrm{Co}, \mathrm{Fe}, \mathrm{K}, \mathrm{Mg}, \mathrm{Mn}, \mathrm{Pb}$ e $\mathrm{Na}$, resultantes da contaminação ocorrida no período de processamento. Até a própolis pode ser contaminada, com tintas, jornais entre outros materiais.

\section{Conclusão}

O monitoramento de resíduos de elementos-traço no mel auxilia na avaliação do potencial de risco desses produtos à saúde do consumidor e fornece informações sobre as características do ambiente de colheita e de suas vizinhanças. Na Alemanha, utiliza-se a análise de traços do mel para verificar a qualidade do ar. Em diversos lugares, o mel já vem sendo inserido na cidade, pois, além de ter um sabor diferenciado devido à variedade de plantas na cidade, o mel produzido no campo pode conter resíduos de agrotóxicos, metais pesados e transgênicos. Assim, é importante que as colmeias não estejam localizadas próximas a áreas onde há grande circulação de automóveis, pois, nesses lugares, pode ocorrer a circulação de grandes quantidades de poluentes provenientes dos escapamentos. Das amostras de mel analisadas, três apresentaram a concentração do elemento chumbo $(\mathrm{Pb})$, fora dos limites estabelecidos pela legislação brasileira (Tabela 1). É necessário que os apicultores reforcem a atenção quanto aos vínculos de contaminação ocorridos em todo o ambiente visitado pelas abelhas. $\mathrm{O}$ mel comercializado deve apresentar níveis reduzidos de metais, principalmente, dos metais pesados. Em seres humanos, o órgão mais afetado pela toxicidade do chumbo, é o sistema nervoso central, podendo até causar sintomas psiquiátricos e prejudicar a destreza manual. Dessa forma, é importante a garantia, ao consumidor, de um produto de qualidade, e livre de contaminações com esses elementos.

\section{Agradecimentos}

Agradecemos à Universidade Estadual do Paraná - Unespar/Campus Paranavaí pelo apoio na realização deste trabalho, e à Fundação Araucária pelo financiamento da pesquisa.

\section{Referências}

ALBUQUERQUE, T. C. S. Adubação mineral da videira. In: FEIRA NACIONAL DA AGRICULTURA IRRIGADA-FENAGRI, 2004, Petrolina. Minicursos: apostilas. Petrolina: Embrapa Semi-Árido, 2004. CD-ROM.

BERTOLDI, F.C.; REIS, V.D.A.; GONZAGA, L.V.; FETT, R.; CONGRO, C.R. Mel silvestre: qualidade para a valorização e a competitividade da apicultura no Pantanal.

Boletim de Pesquisa e Desenvolvimento. Embrapa: Pantanal, 2010.

BRASIL. Ministério da Agricultura, Pecuária e Abastecimento. Instrução Normativa 14/20009. Plano de Controle de Resíduos e Contaminantes em mel. Diário Oficial da União, Brasília, DF, 25 mai. 2009. 
BRASIL. Ministério da Saúde. Decreto $n^{\circ}$ 55.871/1965. Modifica o Decreto $\mathrm{n}^{\circ}$ 50.040/1961, referente às normas reguladoras do emprego de aditivos para alimentos. Diário oficial da União, Brasília, DF, 9 abr. 1965.

BRASIL. Portaria 11/1987. Determina o limite máximo de tolerância de cromo no produto a ser consumindo que não represente risco à saúde. Diário Oficial da União, Brasília, DF, 20 mai. 1987.

BRASIL. Portaria 685/1998. Aprova do Regulamento Técnico: princípios gerais para o estabelecimento de níveis máximos de contaminantes químicos em alimentos. Diário Oficial da União, Brasília, DF, 17 ago. 1998.

BURBURE, C.; BUCHET, J, P. LEROYER, A.; NISSE, C.; HAGUENOER, J. M.; MUTTI, A.; SMERHOVSKY, Z.; CIKRT, M.; TRZCINKA-OCHOCKA, M.; RAZNIEWSKA, G.;JAKUBOWSKI,M.; BERNARD, A. Renal and neurologic effects of cadmium, lead, mercury, and arsenic in children: evidence of early effects and multiple interactions at environmental exposure levels. Environmental Health Perspectives, Cary, NC, v.114, n.4, p.584-590, 2006.

CELECHOVSKÁ, O.; VORLOVÁ, L. Groupsofhoney - Physico chemical properties and heavy metais. Acta Veterinaria Brno, v. 70, p. 91-95. 2001.

CONTI,M.E.; FINOIA, M.G.; FONTANA, L.; MELE, G.; BOTRÈ, F.; IAVICOLI,I. Argentine honey son the basis oftheir mineral contentand some typical quality parameters. Chemistry Central Journal 2014. Disponível em: $<$ http://journal.chemistrycentral.com/ content/8/1/44>. Acesso em: 5 jan. 2015

EPIFÂNIO, A. F. R. P. Determinação de metais pesados em mel nacional por espectrometria de absorção atômica. 2012. 63 f. Dissertação (Mestrado em Segurança Alimentar) - Universidade Técnica de Lisboa - Faculdade de Medicina Veterinária, Portugal, 2012.

HERNÁNDEZ, O.M.; FRAGA,J.M. G.; AI JIMENEZ, A. I.;JIMENEZ, F.; ARIAS, J. J. Characterization of honey from the Canary Islands: determination of the mineral content by atomic absorption spectrophotometry. Food Chemistry.v. 93.p. 449-458.2005.

MEURER, E. J. Fundamentos de Química do Solo. 2. ed. Porto Alegre: Gênesis, 2004. 290 p.

OLIVEIRA, L. F. C.; CASTRO. M. L. L.; RODRIGUES, C.; BORGES, J. D. Isotermas de sorção de metais pesados em solos do cerrado de Goiás. Revista Brasileira de Engenharia Agrícola e Ambiental. v.14, n.7, p.776-782, Campina Grande, PB, 2010. 
PORRINI, C.; SABATINI, A. G.; GIROTTI, S.; GHINI, S.; MEDRZYCKI, P.; GRILLENZONI, F.; BORTOLOTTI, L.; GATTAVECCHIA, E.; CELLI, G. Honey bees and bee products as monitors of the environmental contamination. Apiacta. v. 38, p. 63-70, 2003.

RAEYMAEKERS, B. A prospective biomonitoring campaign with honey bees in a district of Upper-Bavaria (Germany). Environmental Monitoring and Assessment, v. 116, p. 233-243, 2006.

RIBEIRO, R. O.R. Elementos traços em méis de abelhas (apis mellifera) do Estado do Rio de Janeiro, Brasil: a influência da sazonalidade. 106 f. Dissertação (Mestrado em Higiene Veterinária), Universidade Federal Fluminense, 2010.

RISSATO, S.R.; GALHIANE, M.S.; KNOLL, F.R.N.; ANDRADE, R.M.B.; ALMEIDA, M.V.Método multirresíduo para monitoramento da contaminação ambiental de pesticidas na região de Bauru (SP) usando o mel de abelhas como bioindicador. Quím. Nova. v.29, n.5, p.950-955, 2006.

SANTOS, J. S.; SANTOS, N. S.; SANTOS, N. L. P.; SANTOS, S. N.; LACERDA, J. J. J. Honey classification from semi-arid, Atlantic and trasitional forest zones in Bahia, Brazil. Journal Brazilian Chemistry Society, v. 19, n. 3, p. 502-508, 2008.

SODRÉ, G. S.; MARCHINI, L.C.; ZUCCHI, O. L.A.D.; NASCIMENTO FILHO, V. F.; OTSUK, I.P.; MORETI, A. C. C. C. Determination of chemical elements in Africanized Apis mellifera (Hymenoptera: Apidae) honey samples from the State of Piauí, Brazil. Química Nova, v. 30, n. 4, p. 920-924, 2007.

TSUTIYA, M. T. Metais pesados: o principal fator limitante para o uso agrícola de biossólidos das estações de tratamento de esgotos. In: Congresso Brasileiro de Engenharia Sanitária e Ambiental, 20, 1999. Rio de Janeiro, RJ.Anais... Rio de Janeiro: ABES, 1999. p.762-770. 